\title{
Accidental failure to administer an analgesic
}

The IACUC, veterinary staff and Office of Research Compliance at Great Eastern University conscientiously tried to assure that all animals received the painrelieving drugs that were approved by the IACUC. Nevertheless, mistakes did happen occasionally, such as when one lab technician erroneously believed that another technician was administering a once-daily dose of analgesic to a dog, with the result that the dog did not receive its pain relief at all. The error was discovered the next day and reported to the IACUC. The correct schedule of providing analgesia was reinitiated, with the laboratory manager clarifying work assignments for the technicians.

An IACUC subcommittee investigated the incident and determined that it was an unfortunate but unintentional error in a laboratory that had no previous deviations from its approved protocols. No suspension or other sanction was deemed necessary. At the next IACUC meeting, the subcommittee's report of the episode was discussed, approved and entered into the minutes. The Office of Laboratory Animal Welfare was informed of the incident and its resolution.

When it came time to submit the US Department of Agriculture (USDA) annual report, the IACUC administrator placed the dog in column D (pain or distress alleviated by drugs). The IACUC chair questioned this placement, believing that the dog should be in column $\mathrm{E}$ (pain or distress not alleviated by drugs) because of the prolonged time that had passed before analgesia was once again provided. But the administrator stated that using column $\mathrm{D}$ was in line with a previous clarification from the USDA ${ }^{1}$ and, in addition, that column $\mathrm{E}$ is reserved for animals for which pain- or distress-alleviating drugs are not used because of experimental needs. In this case there was no experimental need to withhold drugs, which the administrator said supported the use of column D.

Do you think that the IACUC administrator's explanation and actions were correct, or was the IACUC chairman right? Would you have taken any actions that were not taken by the Great Eastern IACUC?

1. Brown, P. \& Gipson, C. A word from OLAW and USDA. Lab Anim. (NY) 38, 347 (2009).

\section{RESPONSE}

\section{Missed analgesia}

\section{Patricia N. Coan, DVM, PhD, DACLAM, Joleen K. Adams, DVM \& Dana Glass-Mattie, DVM}

We feel that the IACUC administrator was correct: the dog's category of use should be D. A similar situation a few years ago included clarification from the Office of Laboratory Animal Welfare (OLAW) and USDA verifying that category $D$ would be the correct pain category ${ }^{1}$. Unfortunately, the procedures done on the animal and the animal's condition were not presented in this scenario. Nevertheless, the dog did not receive the daily dose of analgesia that should have been provided in accordance with the IACUC-approved protocol. Great Eastern University was correct in forming a subcommittee to evaluate the incident. The Animal Welfare Act and Regulations define a painful procedure as "any procedure that would reasonably be expected to cause more than slight or momentary pain or distress in a human being to which that procedure is applied, that is, pain in excess of that caused by injections or other minor procedures" 2 . The animal did not receive its daily dose of analgesia, and hence the veterinary medical care that was provided was inadequate, in violation of USDA Animal Care Resource Guide Policy \#3: Veterinary Care and Policy \#11: Painful Procedures ${ }^{3}$. Policy 3 was violated in that adequate post-procedural care was not provided and the animal did not receive its once-daily analgesic dose. Policy 11 was violated in that the post-procedural analgesic regimen described in the IACUCapproved protocol was not followed. Policy 11 states that " $[\mathrm{a}]$ nimals exhibiting signs of pain, discomfort, or distress such as weight loss, decreased appetite, abnormal activity level, adverse reactions to touching inoculated areas, open sores/necrotic skin lesions, abscesses, lameness, conjunctivitis, corneal edema, and photophobia are expected to receive appropriate relief unless written scientific justification is provided in the animal activity proposal and approved by the IACUC"3.

The USDA annual report form indicates that category E should include the "number of animals upon which teaching, experiments, research, surgery or tests were conducted involving accompanying pain or distress to the animals and for which the use of appropriate anesthetic, analgesic or tranquilizing drugs would have adversely affected the procedures, results, or interpretation of the teaching, research, experiments, surgery, or tests." Thus, we feel that the dog should not be placed in category E, because analgesics were withheld as a result of human error and not for scientific reasons. There are two areas of concern: the lack of adequate veterinary care and training of the technical staff. We would have required the technical staff members to receive additional training to ensure they understand the importance of reading and following the IACUC-approved protocol, documenting all procedures and treatments in the animal's medical record and notifying the attending veterinarian of any questions or problems.

1. Brown, P. \& Gipson, C. A word from OLAW and USDA. Lab Anim. (NY) 38, 347 (2009).

2. Animal Welfare Regulations. 9 CFR. Chapter 1 Subchapter A,1.1 Definitions.

3. US Department of Agriculture. Animal Care Policy Manual (USDA, Riverdale, MD, 2014). 